\title{
Deep-Trench Structure of Carbon-Fiber-Reinforced Thermoplastics (CFRTP) by Laser Cutting with a Single-Mode IR Fiber Laser
}

\author{
Hiroyuki Niino*, Yoshihisa Harada*, and Akira Fujisaki** \\ * National Institute of Advanced Industrial Science and Technology (AIST), Tsukuba, Japan \\ ** Furukawa Electric Co., Ltd., Chiba, Japan \\ E-mail: niino.hiro@aist.go.jp
}

\begin{abstract}
We report deep-trench microstructures fabricated on carbon-fiber-reinforced thermoplastic (CFRTP; a CFRP containing thermoplastic resin). Well-defined laser cutting free of debris around the trench was achieved for various types of CFRTP plates by single-mode laser irradiation with a fast beam galvanometer scanner using a multiple-scan-pass method. The microfabrication of a CFRTP containing pitch-type carbon fibers and a polycarbonate resin, performed by 600-scan-pass irradiation with a cw kilowatt single-mode IR fiber laser (average power: $1 \mathrm{~kW}$ ), yielded a trench microstructure with a depth of $18.2 \mathrm{~mm}$ and an opening width of $0.32 \mathrm{~mm}$. The laser-induced damage in the samples was analyzed by microscopic X-ray computed tomography. Laser cutting by high-speed beam scanning resulted in a clean top and excellent sidewall quality, along with a negligible heataffected zone.
\end{abstract}

DOI: $10.2961 /$ jlmn.2016.03.0016

Keywords: laser micromachining, deep-trench microstructures, cw single-mode IR fiber laser, CFRTP composite, microscopic X-ray computed tomography

\section{Introduction}

Laser cutting of carbon-fiber-reinforced plastics (CFRPs) with a high-power laser enables well-defined microfabrication without debris around grooves [1]. This cutting process yields high-precision cuts with narrow kerfs where complex contours demand precise, fast and forcefree processing $[2,3]$. To explore the possibility of 3D laser processing, a CFRP was cut into a three-dimensional molded sample using a five-axis laser cutting machine [4]. Single-mode fiber lasers are expected to provide narrow cutting kerfs with high aspect ratios for CFRPs [5], as their beams can be tightly focused with a longer depth-of-focus (DOF) than multi-mode lasers.

In this paper, we report deep-trench microstructures fabricated by laser cutting of carbon-fiber-reinforced thermoplastics (CFRTPs), which are composite materials of thermoplastic resin matrices and carbon fibers, using a cw single-mode fiber laser $(\lambda=1084 \mathrm{~nm})$. Well-defined cutting with a high aspect ratio was achieved for CFRTPs by irradiation with a single-mode laser with a fast beam galvanometer scanner using a multiple-scan-pass method [1, 4]. The deep-trench microstructure in the samples was characterized by X-ray computed tomography (X-CT) to determine the heat-affected zone (HAZ).

\section{Experimental}

The output of a near-IR cw laser (Furukawa Electric, Yb-doped fiber laser, $\lambda=1084 \mathrm{~nm}$, single-mode $\left(\mathrm{M}^{2}<1.1\right.$, BPP: $0.345 \mathrm{~mm}$ mrad, fiber core diameter: $12 \mu \mathrm{m}), 1 \mathrm{~kW}$ average power) [6] was scanned on the sample surface by single-line irradiation of the multiple-scan-pass method with a galvanometer scanner in ambient air (scanning speed: 3.6 and $2.3 \mathrm{~m} \mathrm{~s}^{-1}$, without assist gas) [1, 4]. The beam was incident normal to the sample surface and fo- cused with an f-theta lens (Showa Optronics Co., Ltd., FT300/5-1080F, non-telecentric lens, $\mathrm{f}=306 \mathrm{~mm}$, calculated focal diameter of laser beam on sample surface: 120 $\mu \mathrm{m})$. The time interval (breaking time) between the scanpass irradiations was set to $5 \mathrm{~s}$ to avoid an increase in temperature due to heat accumulation on the sample.

To determine the laser beam profile in the vertical direction, laser engraving of the CFRTP plate was performed with a single sweep at a cw laser power of $130 \mathrm{~W}$. The sample plate was irradiated at an incident angle of $45^{\circ}$. The sweep length of the laser beam for this verification was around $50 \mathrm{~mm}$ on the sample surface.

3-mm-thick and 30-mm-thick CFRTP samples, prepared by compression molding, were employed for the laser cutting experiments. CFRTP-PC consists of a chopped pitch-type carbon fiber ( $\mathrm{Cf}=30 \mathrm{wt} \%)$ and polycarbonate resin (Mitsubishi Engineering-Plastics Corp.; Iupilon, resin melting point: $\mathrm{Tm}=220-230^{\circ} \mathrm{C}$, resin pyrolysis in air: $470^{\circ} \mathrm{C}$ ) [7, 8]. In addition, two other types of CFRTP plate samples were employed for the laser cutting. Both prepared by injection molding, these samples were a chopped PANtype carbon fiber $(\mathrm{Cf}=30 \mathrm{wt} \%)$ in ABS resin (ABS; copolymer of acrylonitrile-butadiene-styrene, Toray Industries Inc.) and in PA6 resin (polyamide 6 (nylon 6), Toray Industries Inc.).

High-resolution images of the sample surface with a large field-of-view obtained by the image stitching method were captured with an optical microscope (Keyence, VHX1000). The deep-trench microstructure in the samples was determined with a microscopic X-CT system (Yamato Sci-

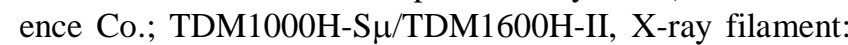
$\mathrm{LaB}_{6}, \mathrm{X}$-ray spot diameter: $\left.0.8 \mu \mathrm{m}\right)$. 


\section{Results and Discussion}

\subsection{Deep-trench structure formation of CFRTP-PC material by laser cutting}

Figure 1 shows cross-sectional images of the trench structure on a CFRTP-PC sample obtained after cw fiber laser irradiation with an average power of $1 \mathrm{~kW}$ using the multiple-scan-pass method. A deep trench with a depth of $18,200 \mu \mathrm{m}$ was obtained by 600 -multiple-scan-pass irradiation (Fig. 1(a)). The kerf width of the trench at the laser incident surface was $320 \mu \mathrm{m}$. The internal kerf in the middle region of the trench was around $150 \mu \mathrm{m}$, as shown in Fig. 1(b). The focal position of the laser beam during the laser irradiation was fixed at $10 \mathrm{~mm}$ beneath the laser incident surface.

Figure 1(c) shows a trench with a depth of $14,800 \mu \mathrm{m}$ obtained after 300-pass irradiation. The kerf width of the trench at the laser incident surface was around $300 \mu \mathrm{m}$, while that of the internal kerf in the bottom region of the trench was around $100 \mu \mathrm{m}$. In addition, the HAZ, as analyzed by X-CT measurement, was 30-50 $\mu \mathrm{m}$ at the internal wall of the groove, as shown in Fig. 1(d). These results indicate that irradiation with a single-mode laser by the multiple-scan-pass method is effective for fabricating a deep-trench microstructure on the CFRTP-PC material.
3.2 Laser engraving of the CFRTP plate by singlemode laser irradiation with an incident angle of $45^{\circ}$

The kerf width of the trench on the sample surface was monitored by laser engraving of the CFRTP plate with an incident angle of $45^{\circ}$. Figure 2 shows the optical micrograph of a large field-of-view on the CFRTP-PC plate surface after a single sweep of the laser beam (average power: $130 \mathrm{~W})$. The laser beam was swept across the sample surface at a speed of $5.1 \mathrm{~m} \mathrm{~s}^{-1}\left(5.1 \mathrm{~m} \mathrm{~s}^{-1} \approx 3.6 \mathrm{~m} \mathrm{~s}^{-1} \times \sqrt{2}\right)$. A track on the CFRTP-PC surface by the laser engraving in Fig. 2 reflects the laser beam profile in the vertical direction. At the focal point, the CFRTP-PC plate was etched by a track width of $0.20 \mathrm{~mm}$. An etched width of $0.4 \mathrm{~mm}$ was observed at a distance of $14 \mathrm{~mm}$ from the focal position. This indicates that laser cutting with a narrow kerf and high aspect ratio took place within a vertical depth of $\pm 10 \mathrm{~mm}$ from the focal position $( \pm 10 \approx \pm 14 / \sqrt{2})$. The above results are also consistent with the long depth-of-focus of the single-mode beam, which is an essential property for microfabrication.

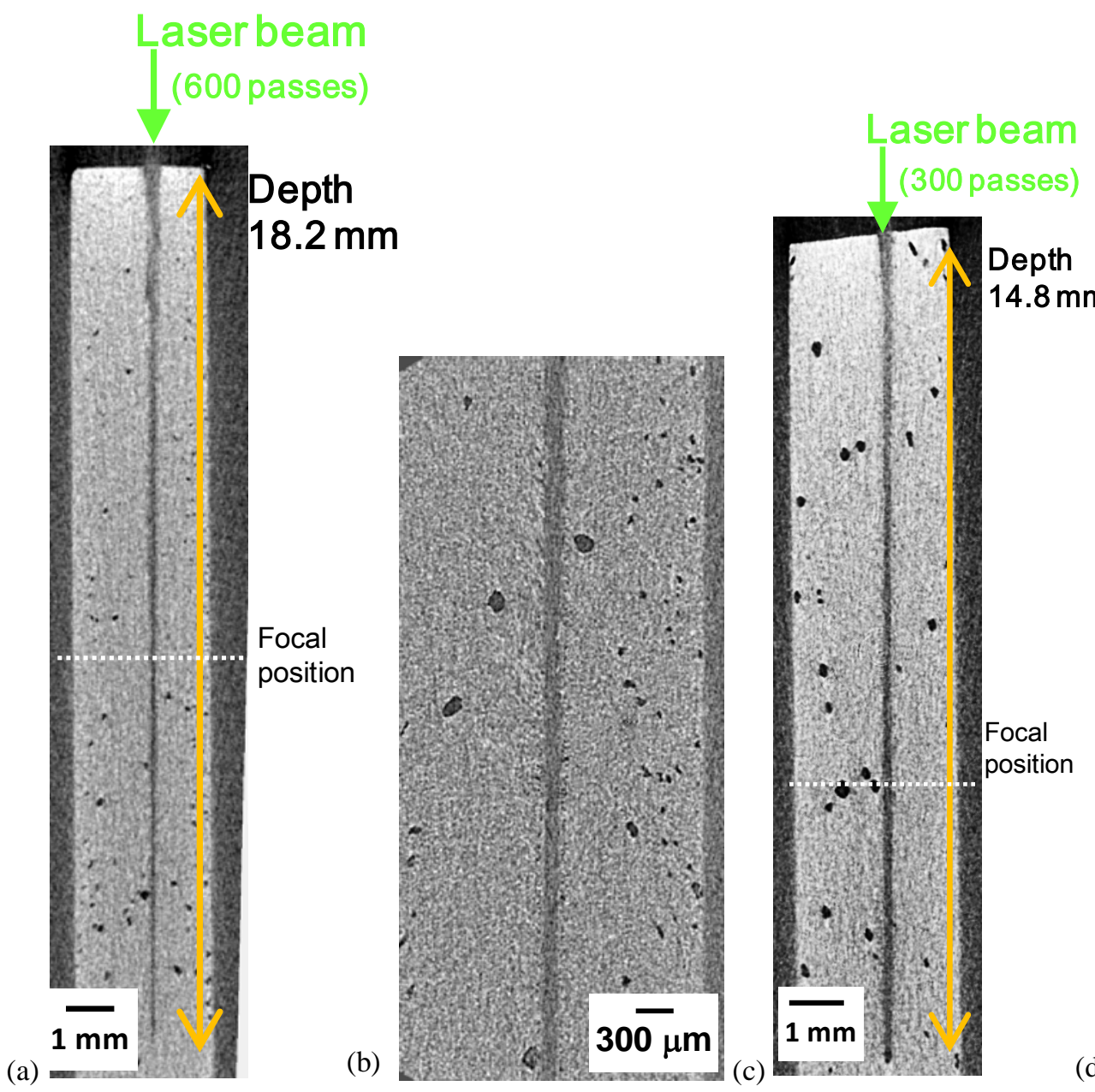
Fig. 1 X-CT image of the 30-mm-thick CFRTP-PC plate cut by cw 1-kW single-mode fiber laser irradiation using the multiple-scan-pass
method. Laser scanning speed was set at $3.6 \mathrm{~m} \mathrm{~s}^{-1}$. (a) Cross-section of the trench fabricated by 600 -multiple-scan-pass irradiation (the deepest area), (b) close-up near the middle of the trench by 600 passes, (c) cross-section of the trench by 300 passes (the deepest area), and (d) close-up of the lower part of the trench by 300 passes. 
3.3 Laser cutting properties of CFRTP-PC

\subsubsection{Laser cutting properties of 3-mm-thick sample}

The cross-sectional images of the CFRTP-PC sample taken after $\mathrm{cw}$ laser irradiation at normal incidence by the multiple-scan-pass method are shown in Fig. 3. The laser scanning speed was set at $2.3 \mathrm{~m} \mathrm{~s}^{-1}$ or $3.6 \mathrm{~m} \mathrm{~s}^{-1}$. In addition, observation normal to the grooves (observation II) verified that the groove depth remained uniform until the plate was cut completely. The kerf width of the groove at the laser incident surface was estimated to be $200 \mu \mathrm{m}$, and that of the groove in the bulk region was also around $200 \mu \mathrm{m}$. The $\mathrm{HAZ}$ at the internal wall of the groove was estimated to be $30 \mu \mathrm{m}$ based on a X-CT measurement.
The depth of the cut grooves increased in proportion to the number of passes of cw 1-kW laser radiation (Fig. 4). Compared to irradiation with a multi-mode fiber laser (fiber core diameter: $50 \mu \mathrm{m} ; 42$ passes at a scanning speed of $2.3 \mathrm{~m} \mathrm{~s}^{-1}$ ) [9], the cutting speed by single-mode laser irradiation was 2.3 times higher.

These results indicate that the etching rate of the CFRTP-PC samples by repeated irradiation with the $\mathrm{cw}$ fiber laser is constant throughout the cutting process of the 3-mm-thick plate and that the depth of grooves in CFRTPPC samples can be precisely controlled by varying the scan speed and the number of passes.

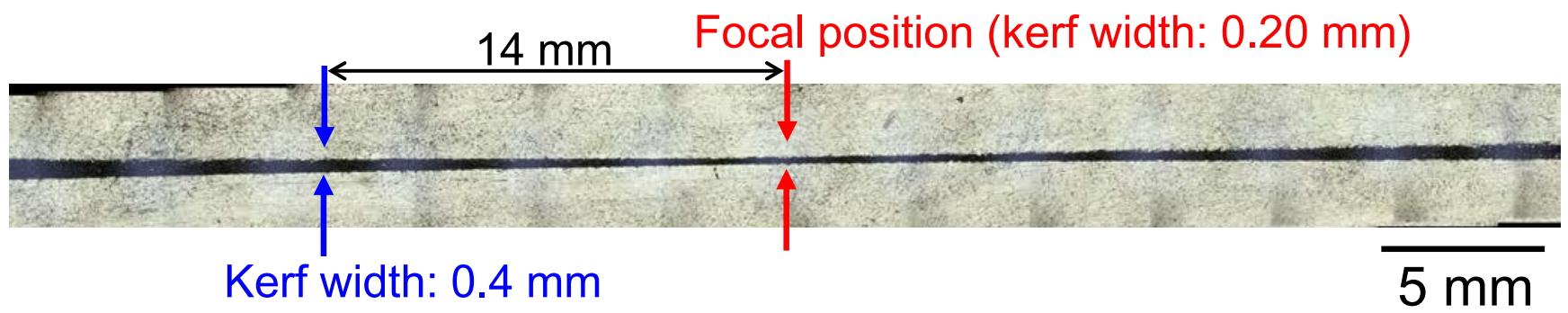

Fig. 2 Optical micrograph with a large field-of-view on the CFRTP-PC plate surface. The CFRTP-PC plate was tilted by $45^{\circ}$ with respect to the incident direction of the single-mode laser (scanning: single sweep with a speed of $5.1 \mathrm{~m} \mathrm{~s}^{-1}$, cw laser power: $130 \mathrm{~W}$ ). The focal position on the plate surface was on the center axis of the scanner.

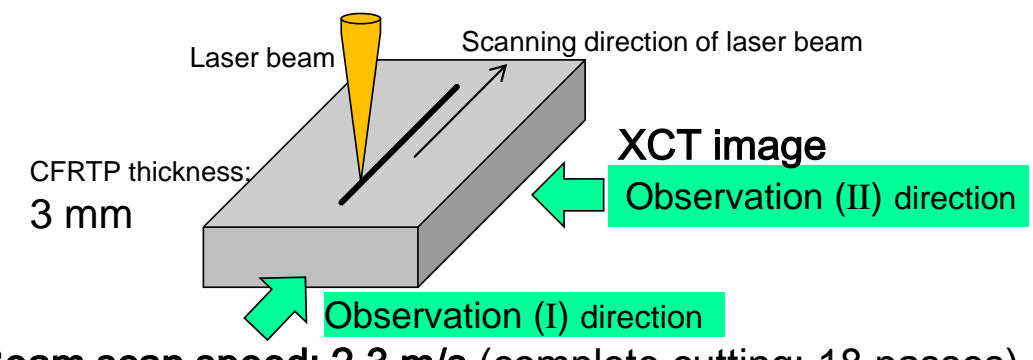

Beam scan speed: $2.3 \mathrm{~m} / \mathrm{s}$ (complete cutting: 18 passes)

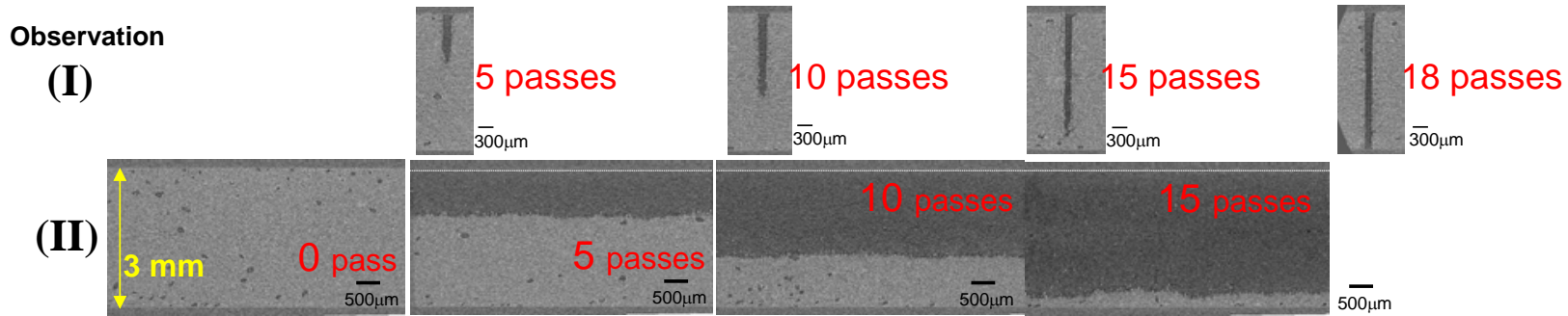

Beam scan speed: $3.6 \mathrm{~m} / \mathrm{s}$ (complete cutting: 31 passes)

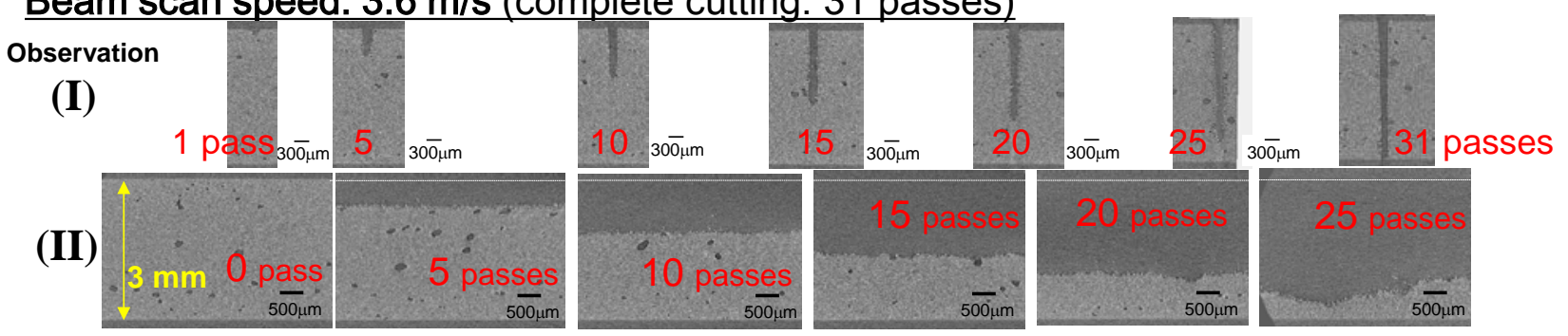

Fig. 3 Cross-sectional X-CT images of the 3-mm-thick CFRTP-PC samples measured from two different observation directions ((I) and (II)). The CFRTP-PC samples were cut by cw single-mode fiber laser irradiation at an average power of $1 \mathrm{~kW}$ and scanning speeds of 2.3 and $3.6 \mathrm{~m} \mathrm{~s}^{-1}$. 


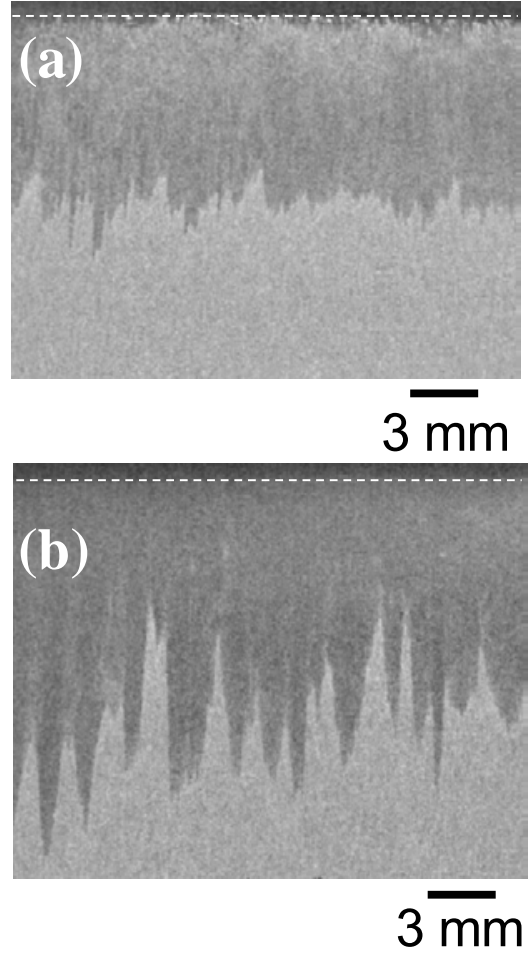

Fig. 7 Cross-sectional X-CT images of the 30-mm-thick CFRTP samples after laser irradiation (1 kW (cw), $3.6 \mathrm{~m} \mathrm{~s}^{-1}$ ); (a) CFRTPABS after 300 passes, (b) CFRTP-PA after 600 passes.

\section{Summary}

We have performed micro-cutting of CFRTP in ambient air by multiple-scan-pass irradiation with a cw near-IR laser. The beam properties of the single-mode laser have a significant effect on the depth of the microtrench on the CFRTP plate. Single-mode laser irradiation with a large DOF of the focused beam afforded a trench structure with a depth of more than $10 \mathrm{~mm}$ and a submillimeter narrow kerf. Laser cutting by high-speed beam scanning with a galvanometer exhibits a clean top and excellent sidewall quality along with a thickness of tens of microns for the HAZ region in the trench.

\section{Acknowledgments}

This work was supported in part by the national project of "Advanced Laser and Processing Technology for Nextgeneration Materials Project” (High-power Pulsed Fiber Laser and Processing Technology Project) launched in 2010FY (period: 5 years) in Industrial Technology Center of NEDO.

\section{References}

[1] H. Niino, Y. Harada, K. Anzai, M. Matsushita, K. Furukawa, M. Nishino, A. Fujisaki, and T. Miyato: JLMN-Journal of Laser Micro/Nanoengineering, 11, (2016) 104.

[2] K. Sugioka, M. Meunier, and A. Piqué, (Eds.): "Laser Precision Microfabrication, Springer Series in Materials Science, Vol. 135”, (Springer-Verlag, Berlin \& Heidelberg, 2010).

[3] D. Bauerle: "Laser Processing and Chemistry (4th Ed.)”, (Springer-Verlag, Berlin \& Heidelberg, 2011).

[4] H. Niino, Y. Harada, K. Anzai, M. Aoyama, M. Matsushita, K. Furukawa, M. Nishino, A.Fujisaki, T. Miyato, and T. Kayahara: Proc. of SPIE, 9353, (2015) p.935303.

[5] R. Staehr, S. Bluemel, P. Jaeschke, O. Suttmann, and L. Overmeyer: Journal of Laser Applications, 28,(2016) 022203.

[6] T. Miyato, T. Kayahara, A. Fujisaki, K. Furukawa, M. Matsushita, M. Muramatsu, Y. Harada, and H. Niino: Proc. of SPIE, 8963, (2014) p.89630W.

[7] H. Niino, Y. Kawaguchi, T. Sato, A. Narazaki, R. Kurosaki, M. Muramatsu, Y. Harada, K. Wakabayashi, T. Nagashima, Z. Kase, M. Matsushita, K. Furukawa, and M. Nishino: JLMN-Journal of Laser Micro/Nanoengineering, 9, (2014) 180.

[8] Mitsubishi Engineering Plastics: Iupilon; Product Information and Material Safety Data Sheet (MSDS); URL http://www.m-ep.co.jp/en/product/brand/

[9] H. Niino, Y. Kawaguchi, T. Sato, A. Narazaki, R. Kurosaki, M. Muramatsu, Y. Harada, K. Wakabayashi, T. Nagashima, Z. Kase, M. Matsushita, K. Furukawa, and M. Nishino: JLMN-Journal of Laser Micro/Nanoengineering, 9, (2014) 180.

(Received: May 29, 2016, Accepted: September 27, 2016) 